\title{
INEQUALITIES OF JENSEN'S TYPE FOR GENERALIZED $k$-g-FRACTIONAL INTEGRALS
}

\author{
SILVESTRU SEVER DRAGOMIR
}

\begin{abstract}
In this paper we establish some inequalities of Jensen and Hermite-Hadamard type for the $k$ - $g$-fractional integrals of convex functions defined an interval $[a, b]$. Some examples for the generalized left- and right-sided Riemann-Liouville fractional integrals of a function $f$ with respect to another function $g$ on $[a, b]$ and for classical RiemannLiouville fractional integrals are also given.
\end{abstract}

\section{Introduction}

The following integral inequality

$$
f\left(\frac{a+b}{2}\right) \leq \frac{1}{b-a} \int_{a}^{b} f(t) d t \leq \frac{f(a)+f(b)}{2},
$$

which holds for any convex function $f:[a, b] \rightarrow \mathbb{R}$, is well known in the literature as the Hermite-Hadamard inequality.

There is an extensive amount of literature devoted to this simple and nice result which has many applications in Theory of Special Means and in Information Theory for divergence measures, from which we would like to refer the reader to the monograph [22], the recent survey paper [15] and the references therein.

Let $f:[a, b] \rightarrow \mathbb{C}$ be a complex valued Lebesgue integrable function on the real interval $[a, b]$. The Riemann-Liouville fractional integrals are defined for $\alpha>0$ by

$$
J_{a+}^{\alpha} f(x)=\frac{1}{\Gamma(\alpha)} \int_{a}^{x}(x-t)^{\alpha-1} f(t) d t
$$

for $a<x \leq b$ and

$$
J_{b-}^{\alpha} f(x)=\frac{1}{\Gamma(\alpha)} \int_{x}^{b}(t-x)^{\alpha-1} f(t) d t
$$

Received December 20, 2017, accpted January 22, 2018.

2010 Mathematics Subject Classification. 26D15, 26D10, 26D07, 26 A33.

Key words and phrases. Generalized Riemann-Liouville fractional integrals, Hadamard fractional integrals, Convex functions, Jensen type inequalities, Hermite-Hadamard type inequalities. 
for $a \leq x<b$, where $\Gamma$ is the Gamma function. For $\alpha=0$, they are defined as

$$
J_{a+}^{0} f(x)=J_{b-}^{0} f(x)=f(x) \text { for } x \in(a, b) .
$$

In the recent paper [16] we obtained the following Hermite-Hadamard type inequalities for convex functions and the Riemann-Liouville fractional integrals

$$
\begin{aligned}
\frac{1}{\alpha+1}\left[\frac{1}{\alpha} f(x)+\frac{f(a)+f(b)}{2}\right] & \geq \frac{1}{2} \Gamma(\alpha)\left[\frac{J_{a+}^{\alpha} f(x)}{(x-a)^{\alpha}}+\frac{J_{b-}^{\alpha} f(x)}{(b-x)^{\alpha}}\right] \\
& \geq \int_{0}^{1}(1-s)^{\alpha-1} f\left(s x+(1-s) \frac{a+b}{2}\right) d s \\
& \geq \frac{1}{\alpha} f\left(\frac{\alpha}{\alpha+1}\left(\frac{x}{\alpha}+\frac{a+b}{2}\right)\right)
\end{aligned}
$$

and

$$
\begin{aligned}
\frac{1}{\alpha+1}\left[f(x)+\frac{1}{\alpha} \frac{f(a)+f(b)}{2}\right] & \geq \frac{1}{2} \Gamma(\alpha)\left[\frac{J_{x-}^{\alpha} f(a)}{(x-a)^{\alpha}}+\frac{J_{x+}^{\alpha} f(b)}{(b-x)^{\alpha}}\right] \\
& \geq \int_{0}^{1} s^{\alpha-1} f\left(s x+(1-s) \frac{a+b}{2}\right) d s \\
& \geq \frac{1}{\alpha} f\left(\frac{\alpha}{\alpha+1}\left(x+\frac{1}{\alpha} \frac{a+b}{2}\right)\right)
\end{aligned}
$$

for any $x \in(a, b)$ and $\alpha>0$.

In order to extend these type of inequalities for more general fractional integrals we need the following preparations.

Assume that the kernel $k$ is defined either on $(0, \infty)$ or on $[0, \infty)$ with complex values and integrable on any finite subinterval. We define the function $K:[0, \infty) \rightarrow \mathbb{C}$ by

$$
K(t):= \begin{cases}\int_{0}^{t} k(s) d s & \text { if } 0<t, \\ 0 & \text { if } t=0 .\end{cases}
$$

As a simple example, if $k(t)=t^{\alpha-1}$ then for $\alpha \in(0,1)$ the function $k$ is defined on $(0, \infty)$ and $K(t):=\frac{1}{\alpha} t^{\alpha}$ for $t \in[0, \infty)$. If $\alpha \geq 1$, then $k$ is defined on $[0, \infty)$ and $K(t):=\frac{1}{\alpha} t^{\alpha}$ for $t \in[0, \infty)$.

Let $g$ be a strictly increasing function on $(a, b)$, having a continuous derivative $g^{\prime}$ on $(a, b)$. For the Lebesgue integrable function $f:(a, b) \rightarrow \mathbb{C}$, we define the $k$-g-left-sided fractional integral of $f$ by

$$
S_{k, g, a+} f(x)=\int_{a}^{x} k(g(x)-g(t)) g^{\prime}(t) f(t) d t, x \in(a, b]
$$

and the $k$-g-right-sided fractional integral of $f$ by

$$
S_{k, g, b-} f(x)=\int_{x}^{b} k(g(t)-g(x)) g^{\prime}(t) f(t) d t, x \in[a, b) .
$$


If we take $k(t)=\frac{1}{\Gamma(\alpha)} t^{\alpha-1}$, where $\Gamma$ is the Gamma function, then

$$
\begin{aligned}
S_{k, g, a+} f(x) & =\frac{1}{\Gamma(\alpha)} \int_{a}^{x}[g(x)-g(t)]^{\alpha-1} g^{\prime}(t) f(t) d t \\
& =: I_{a+, g}^{\alpha} f(x), a<x \leq b
\end{aligned}
$$

and

$$
\begin{aligned}
S_{k, g, b-} f(x) & =\frac{1}{\Gamma(\alpha)} \int_{x}^{b}[g(t)-g(x)]^{\alpha-1} g^{\prime}(t) f(t) d t \\
& =: I_{b-, g}^{\alpha} f(x), a \leq x<b,
\end{aligned}
$$

which are the generalized left-and right-sided Riemann-Liouville fractional integrals of a function $f$ with respect to another function $g$ on $[a, b]$ as defined in [25, p.100].

For $g(t)=t$ in (1.7) we have the classical Riemann-Liouville fractional integrals while for the logarithmic function $g(t)=\ln t$ we have the Hadamard fractional integrals [25, p. 111]

$$
H_{a+}^{\alpha} f(x):=\frac{1}{\Gamma(\alpha)} \int_{a}^{x}\left[\ln \left(\frac{x}{t}\right)\right]^{\alpha-1} \frac{f(t) d t}{t}, 0 \leq a<x \leq b
$$

and

$$
H_{b-}^{\alpha} f(x):=\frac{1}{\Gamma(\alpha)} \int_{x}^{b}\left[\ln \left(\frac{t}{x}\right)\right]^{\alpha-1} \frac{f(t) d t}{t}, 0 \leq a<x<b .
$$

One can consider the function $g(t)=-t^{-1}$ and define the "Harmonic fractional integrals" by

$$
R_{a+}^{\alpha} f(x):=\frac{x^{1-\alpha}}{\Gamma(\alpha)} \int_{a}^{x} \frac{f(t) d t}{(x-t)^{1-\alpha} t^{\alpha+1}}, 0 \leq a<x \leq b
$$

and

$$
R_{b-}^{\alpha} f(x):=\frac{x^{1-\alpha}}{\Gamma(\alpha)} \int_{x}^{b} \frac{f(t) d t}{(t-x)^{1-\alpha} t^{\alpha+1}}, 0 \leq a<x<b .
$$

Also, for $g(t)=\exp (\beta t), \beta>0$, we can consider the " $\beta$-Exponential fractional integrals"

$$
E_{a+, \beta}^{\alpha} f(x):=\frac{\beta}{\Gamma(\alpha)} \int_{a}^{x}[\exp (\beta x)-\exp (\beta t)]^{\alpha-1} \exp (\beta t) f(t) d t,
$$

for $a<x \leq b$ and

$$
E_{b-, \beta}^{\alpha} f(x):=\frac{\beta}{\Gamma(\alpha)} \int_{x}^{b}[\exp (\beta t)-\exp (\beta x)]^{\alpha-1} \exp (\beta t) f(t) d t,
$$

for $a \leq x<b$.

If we take $g(t)=t$ in (1.4) and (1.5), then we can consider the following $k$-fractional integrals

for $a<x \leq b$ and

$$
S_{k, a+} f(x)=\int_{a}^{x} k(x-t) f(t) d t, x \in(a, b]
$$

$$
S_{k, b-} f(x)=\int_{x}^{b} k(t-x) f(t) d t, x \in[a, b) .
$$


In [28], Raina studied a class of functions defined formally by

$$
\mathscr{F}_{\rho, \lambda}^{\sigma}(x):=\sum_{k=0}^{\infty} \frac{\sigma(k)}{\Gamma(\rho k+\lambda)} x^{k},|x|<R \text {, with } R>0
$$

for $\rho, \lambda>0$ where the coefficients $\sigma(k)$ generate a bounded sequence of positive real numbers. With the help of (1.16), Raina defined the following left-sided fractional integral operator

$$
\mathscr{J}_{\rho, \lambda, a+; w}^{\sigma} f(x):=\int_{a}^{x}(x-t)^{\lambda-1} \mathscr{F}_{\rho, \lambda}^{\sigma}\left(w(x-t)^{\rho}\right) f(t) d t, x>a
$$

where $\rho, \lambda>0, w \in \mathbb{R}$ and $f$ is such that the integral on the right side exists.

In [1], the right-sided fractional operator was also introduced as

$$
\mathscr{J}_{\rho, \lambda, b-; w}^{\sigma} f(x):=\int_{x}^{b}(t-x)_{\rho, \lambda}^{\lambda-1} \mathscr{F}_{\rho, \lambda}^{\sigma}\left(w(t-x)^{\rho}\right) f(t) d t, x<b
$$

where $\rho, \lambda>0, w \in \mathbb{R}$ and $f$ is such that the integral on the right side exists. Several Ostrowski type inequalities were also established.

We observe that for $k(t)=t^{\lambda-1} \mathscr{F}_{\rho, \lambda}^{\sigma}\left(w t^{\rho}\right)$ we re-obtain the definitions of (1.17) and (1.18) from (1.14) and (1.15).

In [26], Kirane and Torebek introduced the following exponential fractional integrals

$$
\mathscr{T}_{a+}^{\alpha} f(x):=\frac{1}{\alpha} \int_{a}^{x} \exp \left\{-\frac{1-\alpha}{\alpha}(x-t)\right\} f(t) d t, x>a
$$

and

$$
\mathscr{T}_{b-}^{\alpha} f(x):=\frac{1}{\alpha} \int_{x}^{b} \exp \left\{-\frac{1-\alpha}{\alpha}(t-x)\right\} f(t) d t, x<b
$$

where $\alpha \in(0,1)$.

We observe that for $k(t)=\frac{1}{\alpha} \exp \left(-\frac{1-\alpha}{\alpha} t\right), t \in \mathbb{R}$ we re-obtain the definitions of (1.19) and (1.20) from (1.14) and (1.15).

Let $g$ be a strictly increasing function on $(a, b)$, having a continuous derivative $g^{\prime}$ on $(a, b)$. We can define the more general exponential fractional integrals

$$
\mathscr{T}_{g, a+}^{\alpha} f(x):=\frac{1}{\alpha} \int_{a}^{x} \exp \left\{-\frac{1-\alpha}{\alpha}(g(x)-g(t))\right\} g^{\prime}(t) f(t) d t, x>a
$$

and

$$
\mathscr{T}_{g, b-}^{\alpha} f(x):=\frac{1}{\alpha} \int_{x}^{b} \exp \left\{-\frac{1-\alpha}{\alpha}(g(t)-g(x))\right\} g^{\prime}(t) f(t) d t, x<b
$$

where $\alpha \in(0,1)$.

Let $g$ be a strictly increasing function on $(a, b)$, having a continuous derivative $g^{\prime}$ on $(a, b)$. Assume that $\alpha>0$. We can also define the logarithmic fractional integrals

$$
\mathscr{L}_{g, a+}^{\alpha} f(x):=\int_{a}^{x}(g(x)-g(t))^{\alpha-1} \ln (g(x)-g(t)) g^{\prime}(t) f(t) d t,
$$


for $0<a<x \leq b$ and

$$
\mathscr{L}_{g, b-}^{\alpha} f(x):=\int_{x}^{b}(g(t)-g(x))^{\alpha-1} \ln (g(t)-g(x)) g^{\prime}(t) f(t) d t,
$$

for $0<a \leq x<b$, where $\alpha>0$. These are obtained from (1.14) and (1.15) for the kernel $k(t)=$ $t^{\alpha-1} \ln t, t>0$.

For $\alpha=1$ we get

$$
\mathscr{L}_{g, a+} f(x):=\int_{a}^{x} \ln (g(x)-g(t)) g^{\prime}(t) f(t) d t, 0<a<x \leq b
$$

and

$$
\mathscr{L}_{g, b-} f(x):=\int_{x}^{b} \ln (g(t)-g(x)) g^{\prime}(t) f(t) d t, 0<a \leq x<b .
$$

For $g(t)=t$, we have the simple forms

$$
\begin{aligned}
& \mathscr{L}_{a+}^{\alpha} f(x):=\int_{a}^{x}(x-t)^{\alpha-1} \ln (x-t) f(t) d t, 0<a<x \leq b, \\
& \mathscr{L}_{b-}^{\alpha} f(x):=\int_{x}^{b}(t-x)^{\alpha-1} \ln (t-x) f(t) d t, 0<a \leq x<b, \\
& \mathscr{L}_{a+} f(x):=\int_{a}^{x} \ln (x-t) f(t) d t, 0<a<x \leq b
\end{aligned}
$$

and

$$
\mathscr{L}_{b-} f(x):=\int_{x}^{b} \ln (t-x) f(t) d t, 0<a \leq x<b .
$$

For several Ostrowski type inequalities for Riemann-Liouville fractional integrals see [2]-[18], [23]-[36] and the references therein.

For $k$ and $g$ as at the beginning of Introduction, we consider the mixed operator

$$
\begin{aligned}
S_{k, g, a+, b-} f(x) & :=\frac{1}{2}\left[S_{k, g, a+} f(x)+S_{k, g, b-} f(x)\right] \\
& =\frac{1}{2}\left[\int_{a}^{x} k(g(x)-g(t)) g^{\prime}(t) f(t) d t+\int_{x}^{b} k(g(t)-g(x)) g^{\prime}(t) f(t) d t\right]
\end{aligned}
$$

for the Lebesgue integrable function $f:(a, b) \rightarrow \mathbb{C}$ and $x \in(a, b)$.

Observe that

and

$$
S_{k, g, x+} f(b)=\int_{x}^{b} k(g(b)-g(t)) g^{\prime}(t) f(t) d t, x \in[a, b)
$$

$$
S_{k, g, x-} f(a)=\int_{a}^{x} k(g(t)-g(a)) g^{\prime}(t) f(t) d t, x \in(a, b] .
$$

We can define also the dual mixed operator

$$
\breve{S}_{k, g, a+, b-} f(x):=\frac{1}{2}\left[S_{k, g, x+} f(b)+S_{k, g, x-} f(a)\right]
$$




$$
=\frac{1}{2}\left[\int_{x}^{b} k(g(b)-g(t)) g^{\prime}(t) f(t) d t+\int_{a}^{x} k(g(t)-g(a)) g^{\prime}(t) f(t) d t\right]
$$

for any $x \in(a, b)$.

In this paper we establish some inequalities of Jensen and Hermite-Hadamard type for the $k$-g-fractional integrals of convex functions defined an interval $[a, b]$. Some examples for the generalized left- and right-sided Riemann-Liouville fractional integrals of a function $f$ with respect to another function $g$ on $[a, b]$ and for classical Riemann-Liouville fractional integrals are also given.

\section{The main results}

We have the following bounds for the operator $S_{k, g, a+, b-} f$ :

Theorem 1. Assume that the kernel $k$ is defined either on $(0, \infty)$ or on $[0, \infty)$ with nonnegative values and integrable on any finite subinterval. Let $g$ be a strictly increasing function on $(a, b)$, having a continuous derivative $g^{\prime}$ on $(a, b)$. If $f:[a, b] \rightarrow \mathbb{R}$ is a convex function, then

$$
\begin{aligned}
\frac{1}{2}[ & K(g(x)-g(a))+K(g(b)-g(x))] \\
& \times f\left(\frac{K(g(x)-g(a)) a+K(g(b)-g(x)) b}{K(g(x)-g(a))+K(g(b)-g(x))}+\frac{\int_{a}^{x} K(g(x)-g(t)) d t-\int_{x}^{b} K(g(t)-g(x)) d t}{K(g(x)-g(a))+K(g(b)-g(x))}\right) \\
\leq & \frac{1}{2}\left[f\left(a+\frac{1}{K(g(x)-g(a))} \int_{a}^{x} K(g(x)-g(t)) d t\right) K(g(x)-g(a))\right. \\
& \left.+f\left(b-\frac{1}{K(g(b)-g(x))} \int_{x}^{b} K(g(t)-g(x)) d t\right) K(g(b)-g(x))\right] \\
\leq & S_{k, g, a+, b-f(x)} \\
\leq & \frac{1}{2}[K(g(x)-g(a)) f(a)+K(g(b)-g(x)) f(b)] \\
& +\frac{1}{2}\left[\frac{f(x)-f(a)}{x-a} \int_{a}^{x} K(g(x)-g(t)) d t-\frac{f(b)-f(x)}{b-x} \int_{x}^{b} K(g(t)-g(x)) d t\right]
\end{aligned}
$$

for $x \in(a, b)$.

Proof. Since $f:[a, b] \rightarrow \mathbb{R}$ is convex, then for $x \in(a, b)$

$$
f(t) \leq \frac{t-a}{x-a} f(x)+\frac{x-t}{x-a} f(a), t \in[a, x]
$$

and

$$
f(t) \leq \frac{t-x}{b-x} f(b)+\frac{b-t}{b-x} f(x), t \in[x, b] .
$$

By (2.2) and (2.3) we have

$$
S_{k, g, a+, b-} f(x)=\frac{1}{2}\left[\int_{a}^{x} k(g(x)-g(t)) g^{\prime}(t) f(t) d t+\int_{x}^{b} k(g(t)-g(x)) g^{\prime}(t) f(t) d t\right]
$$




$$
\begin{aligned}
\leq & \frac{1}{2} \int_{a}^{x} k(g(x)-g(t)) g^{\prime}(t)\left[\frac{t-a}{x-a} f(x)+\frac{x-t}{x-a} f(a)\right] d t \\
& +\frac{1}{2} \int_{x}^{b} k(g(t)-g(x)) g^{\prime}(t)\left[\frac{t-x}{b-x} f(b)+\frac{b-t}{b-x} f(x)\right] d t
\end{aligned}
$$

for $x \in(a, b)$.

Using the chain rule we have

$$
(K(g(x)-g(t)))^{\prime}=-K^{\prime}(g(x)-g(t)) g^{\prime}(t)=-k(g(x)-g(t)) g^{\prime}(t)
$$

for $t \in(a, x)$ and

$$
(K(g(t)-g(x)))^{\prime}=K^{\prime}(g(t)-g(x)) g^{\prime}(t)=k(g(t)-g(x)) g^{\prime}(t)
$$

for $t \in(x, b)$.

Then, integrating by parts, we have

$$
\begin{array}{rl}
\int_{a}^{x} & k(g(x)-g(t)) g^{\prime}(t)\left[\frac{t-a}{x-a} f(x)+\frac{x-t}{x-a} f(a)\right] d t \\
& =-\int_{a}^{x}(K(g(x)-g(t)))^{\prime}\left[\frac{t-a}{x-a} f(x)+\frac{x-t}{x-a} f(a)\right] d t \\
& =-\left[\left.K(g(x)-g(t))\left[\frac{t-a}{x-a} f(x)+\frac{x-t}{x-a} f(a)\right]\right|_{a} ^{x}-\frac{f(x)-f(a)}{x-a} \int_{a}^{x} K(g(x)-g(t)) d t\right] \\
& =K(g(x)-g(a)) f(a)+\frac{f(x)-f(a)}{x-a} \int_{a}^{x} K(g(x)-g(t)) d t
\end{array}
$$

and

$$
\begin{array}{rl}
\int_{x}^{b} & k(g(t)-g(x)) g^{\prime}(t)\left[\frac{t-x}{b-x} f(b)+\frac{b-t}{b-x} f(x)\right] d t \\
& =\int_{x}^{b}(K(g(t)-g(x)))^{\prime}\left[\frac{t-x}{b-x} f(b)+\frac{b-t}{b-x} f(x)\right] d t \\
& =\left.K(g(t)-g(x))\left[\frac{t-x}{b-x} f(b)+\frac{b-t}{b-x} f(x)\right]\right|_{x} ^{b}-\frac{f(b)-f(x)}{b-x} \int_{x}^{b} K(g(t)-g(x)) d t \\
& =K(g(b)-g(x)) f(b)-\frac{f(b)-f(x)}{b-x} \int_{x}^{b} K(g(t)-g(x)) d t
\end{array}
$$

for $x \in(a, b)$.

Therefore by (2.4) we have

$$
\begin{aligned}
S_{k, g, a+, b-} f(x) \leq & \frac{1}{2}\left[\frac{f(x)-f(a)}{x-a} \int_{a}^{x} K(g(x)-g(t)) d t+K(g(x)-g(a)) f(a)\right] \\
& +\frac{1}{2}\left[K(g(b)-g(x)) f(b)-\frac{f(b)-f(x)}{b-x} \int_{x}^{b} K(g(t)-g(x)) d t\right]
\end{aligned}
$$




$$
\begin{aligned}
= & \frac{1}{2}[K(g(x)-g(a)) f(a)+K(g(b)-g(x)) f(b)] \\
& +\frac{1}{2}\left[\frac{f(x)-f(a)}{x-a} \int_{a}^{x} K(g(x)-g(t)) d t-\frac{f(b)-f(x)}{b-x} \int_{x}^{b} K(g(t)-g(x)) d t\right]
\end{aligned}
$$

for $x \in(a, b)$, which proves the third inequality in (2.1).

We use the Jensen inequality in the form

$$
\frac{\int_{c}^{d} w(t) f(t) d t}{\int_{c}^{d} w(t) d t} \geq f\left(\frac{\int_{c}^{d} w(t) t d t}{\int_{c}^{d} w(t) d t}\right)
$$

where $f:[c, d] \rightarrow \mathbb{R}$ is convex and $w(t) \geq 0, t \in[c, d]$ is integrable with $\int_{c}^{d} w(t) d t>0$.

Therefore

$$
\begin{aligned}
& \int_{a}^{x} k(g(x)-g(t)) g^{\prime}(t) f(t) d t \\
& \quad \geq f\left(\frac{\int_{a}^{x} k(g(x)-g(t)) g^{\prime}(t) t d t}{\int_{a}^{x} k(g(x)-g(t)) g^{\prime}(t) d t}\right) \int_{a}^{x} k(g(x)-g(t)) g^{\prime}(t) d t
\end{aligned}
$$

and

$$
\begin{aligned}
& \int_{x}^{b} k(g(t)-g(x)) g^{\prime}(t) f(t) d t \\
& \quad \geq f\left(\frac{\int_{x}^{b} k(g(t)-g(x)) g^{\prime}(t) t d t}{\int_{x}^{b} k(g(t)-g(x)) g^{\prime}(t) d t}\right) \int_{x}^{b} k(g(t)-g(x)) g^{\prime}(t) d t
\end{aligned}
$$

for $x \in(a, b)$.

We have

and

$$
\int_{a}^{x} k(g(x)-g(t)) g^{\prime}(t) d t=-\int_{a}^{x}(K(g(x)-g(t)))^{\prime} d t=K(g(x)-g(a))
$$

$$
\begin{aligned}
\int_{a}^{x} k(g(x)-g(t)) g^{\prime}(t) t d t & =-\int_{a}^{x}(K(g(x)-g(t)))^{\prime} t d t \\
& =-\left[\left.K(g(x)-g(t)) t\right|_{a} ^{x}-\int_{a}^{x} K(g(x)-g(t)) d t\right] \\
& =K(g(x)-g(a)) a+\int_{a}^{x} K(g(x)-g(t)) d t
\end{aligned}
$$

for $x \in(a, b)$.

Also

$$
\int_{x}^{b} k(g(t)-g(x)) g^{\prime}(t) d t=\int_{x}^{b}(K(g(t)-g(x)))^{\prime} d t=K(g(b)-g(x))
$$

and

$$
\int_{x}^{b} k(g(t)-g(x)) g^{\prime}(t) t d t=\int_{x}^{b}(K(g(t)-g(x)))^{\prime} t d t
$$




$$
\begin{aligned}
& =\left.K(g(t)-g(x)) t\right|_{x} ^{b}-\int_{x}^{b} K(g(t)-g(x)) d t \\
& =K(g(b)-g(x)) b-\int_{x}^{b} K(g(t)-g(x)) d t
\end{aligned}
$$

for $x \in(a, b)$.

Then by (2.6) and (2.7) we have

$$
\begin{array}{rl}
\int_{a}^{x} & k(g(x)-g(t)) g^{\prime}(t) f(t) d t \\
& \geq f\left(a+\frac{1}{K(g(x)-g(a))} \int_{a}^{x} K(g(x)-g(t)) d t\right) K(g(x)-g(a))
\end{array}
$$

and

$$
\begin{aligned}
& \int_{x}^{b} k(g(t)-g(x)) g^{\prime}(t) f(t) d t \\
& \quad \geq f\left(b-\frac{1}{K(g(b)-g(x))} \int_{x}^{b} K(g(t)-g(x)) d t\right) K(g(b)-g(x))
\end{aligned}
$$

for $x \in(a, b)$.

Using the inequalities (2.8) and (2.9) we have

$$
\begin{aligned}
S_{k, g, a+, b-} f(x) & =\frac{1}{2}\left[\int_{a}^{x} k(g(x)-g(t)) g^{\prime}(t) f(t) d t+\int_{x}^{b} k(g(t)-g(x)) g^{\prime}(t) f(t) d t\right] \\
& \geq \frac{1}{2} f\left(a+\frac{1}{K(g(x)-g(a))} \int_{a}^{x} K(g(x)-g(t)) d t\right) K(g(x)-g(a)) \\
& +\frac{1}{2} f\left(b-\frac{1}{K(g(b)-g(x))} \int_{x}^{b} K(g(t)-g(x)) d t\right) K(g(b)-g(x)),
\end{aligned}
$$

which proves the second inequality in (2.1).

By the convexity of $f$ we have for $\alpha, \beta \geq 0$ with $\alpha+\beta>0$, that

$$
\frac{\alpha f(c)+\beta f(d)}{\alpha+\beta} \geq f\left(\frac{\alpha c+\beta d}{\alpha+\beta}\right) \text {. }
$$

Then for

and

$$
\alpha=\frac{K(g(x)-g(a))}{2}, \quad \beta=\frac{K(g(b)-g(x))}{2}
$$

$$
\begin{aligned}
& c=a+\frac{1}{K(g(x)-g(a))} \int_{a}^{x} K(g(x)-g(t)) d t, \\
& d=b-\frac{1}{K(g(b)-g(x))} \int_{x}^{b} K(g(t)-g(x)) d t
\end{aligned}
$$

we have

$f\left(a+\frac{1}{K(g(x)-g(a))} \int_{a}^{x} K(g(x)-g(t)) d t\right) \frac{K(g(x)-g(a))}{2}$ 


$$
\begin{aligned}
& +f\left(b-\frac{1}{K(g(b)-g(x))} \int_{x}^{b} K(g(t)-g(x)) d t\right) \frac{K(g(b)-g(x))}{2} \\
\geq & \frac{1}{2}[K(g(x)-g(a))+K(g(b)-g(x))] \\
& \times f\left(\frac{K(g(x)-g(a)) a+K(g(b)-g(x)) b}{K(g(x)-g(a))+K(g(b)-g(x))}+\frac{\int_{a}^{x} K(g(x)-g(t)) d t-\int_{x}^{b} K(g(t)-g(x)) d t}{K(g(x)-g(a))+K(g(b)-g(x))}\right),
\end{aligned}
$$

which proves the first inequality in (2.1).

If $g$ is a function which maps an interval $I$ of the real line to the real numbers, and is both continuous and injective then we can define the $g$-mean of two numbers $a, b \in I$ as

$$
M_{g}(a, b):=g^{-1}\left(\frac{g(a)+g(b)}{2}\right) .
$$

If $I=\mathbb{R}$ and $g(t)=t$ is the identity function, then $M_{g}(a, b)=A(a, b):=\frac{a+b}{2}$, the arithmetic mean. If $I=(0, \infty)$ and $g(t)=\ln t$, then $M_{g}(a, b)=G(a, b):=\sqrt{a b}$, the geometric mean. If $I=(0, \infty)$ and $g(t)=\frac{1}{t}$, then $M_{g}(a, b)=H(a, b):=\frac{2 a b}{a+b}$, the harmonic mean. If $I=(0, \infty)$ and $g(t)=t^{p}, p \neq 0$, then $M_{g}(a, b)=M_{p}(a, b):=\left(\frac{a^{p}+b^{p}}{2}\right)^{1 / p}$, the power mean with exponent $p$. Finally, if $I=\mathbb{R}$ and $g(t)=\exp t$, then

$$
M_{g}(a, b)=\operatorname{LME}(a, b):=\ln \left(\frac{\exp a+\exp b}{2}\right),
$$

the LogMeanExp function.

Corollary 1. With the assumptions of Theorem 1 we have

$$
\begin{aligned}
K( & \left.\frac{g(b)-g(a)}{2}\right) f\left(\frac{a+b}{2}+\frac{\int_{a}^{M_{g}(a, b)} K\left(\frac{g(a)+g(b)}{2}-g(t)\right) d t-\int_{M_{g}(a, b)}^{b} K\left(g(t)-\frac{g(a)+g(b)}{2}\right) d t}{2 K\left(\frac{g(b)-g(a)}{2}\right)}\right) \\
\leq & \frac{1}{2}\left[f\left(a+\frac{1}{K\left(\frac{g(b)-g(a)}{2}\right)} \int_{a}^{M_{g}(a, b)} K\left(\frac{g(a)+g(b)}{2}-g(t)\right) d t\right)\right. \\
& \left.\left.+f\left(b-\frac{1}{K\left(\frac{g(b)-g(a)}{2}\right)} \int_{M_{g}(a, b)}^{b} K\left(g(t)-\frac{g(a)+g(b)}{2}\right) d t\right)\right] K\left(\frac{g(b)-g(a)}{2}\right)\right] \\
\leq & S_{k, g, a+, b-f\left(M_{g}(a, b)\right)} \\
\leq & \frac{1}{2}[f(a)+f(b)] K\left(\frac{g(b)-g(a)}{2}\right) \\
& +\frac{1}{2} \frac{f\left(M_{g}(a, b)\right)-f(a)}{M_{g}(a, b)-a} \int_{a}^{M_{g}(a, b)} K\left(\frac{g(a)+g(b)}{2}-g(t)\right) d t \\
& -\frac{1}{2} \frac{f(b)-f\left(M_{g}(a, b)\right)}{b-M_{g}(a, b)} \int_{M_{g}(a, b)}^{b} K\left(g(t)-\frac{g(a)+g(b)}{2}\right) d t .
\end{aligned}
$$


For the dual operator $\breve{S}_{k, g, a+, b-} f$ we also have the following bounds:

Theorem 2. Assume that the kernel $k$ is defined either on $(0, \infty)$ or on $[0, \infty)$ with nonnegative values and integrable on any finite subinterval. Let $g$ be a strictly increasing function on $(a, b)$, having a continuous derivative $g^{\prime}$ on $(a, b)$. If $f:[a, b] \rightarrow \mathbb{R}$ is a convex function, then

$$
\begin{aligned}
\frac{1}{2}[ & K(g(x)-g(a))+K(g(b)-g(x))] \\
& \times f\left(x+\frac{\int_{x}^{b} K(g(b)-g(t)) d t-\int_{a}^{x} K(g(t)-g(a)) d t}{K(g(x)-g(a))+K(g(b)-g(x))}\right) \\
\leq & \frac{1}{2}\left[f\left(x-\frac{1}{K(g(x)-g(a))} \int_{a}^{x} K(g(t)-g(a)) d t\right) K(g(x)-g(a))\right. \\
& \left.+f\left(x+\frac{1}{K(g(b)-g(x))} \int_{x}^{b} K(g(b)-g(t)) d t\right) K(g(b)-g(x))\right] \\
\leq & \breve{S}_{k, g, a+, b-f(x)} \\
\leq & \frac{1}{2}[K(g(x)-g(a))+K(g(b)-g(x))] f(x) \\
& +\frac{1}{2}\left[\frac{f(b)-f(x)}{b-x} \int_{x}^{b} K(g(b)-g(t)) d t-\frac{f(x)-f(a)}{x-a} \int_{a}^{x} K(g(t)-g(a)) d t\right]
\end{aligned}
$$

for $x \in(a, b)$.

Proof. Using (2.2) and (2.3) we have

$$
\begin{aligned}
\breve{S}_{k, g, a+, b-} f(x)= & \frac{1}{2} \int_{a}^{x} k(g(t)-g(a)) g^{\prime}(t) f(t) d t+\frac{1}{2} \int_{x}^{b} k(g(b)-g(t)) g^{\prime}(t) f(t) d t \\
\leq & \frac{1}{2} \int_{a}^{x} k(g(t)-g(a)) g^{\prime}(t)\left[\frac{t-a}{x-a} f(x)+\frac{x-t}{x-a} f(a)\right] d t \\
& +\frac{1}{2} \int_{x}^{b} k(g(b)-g(t)) g^{\prime}(t)\left[\frac{t-x}{b-x} f(b)+\frac{b-t}{b-x} f(x)\right] d t
\end{aligned}
$$

for $x \in(a, b)$.

Using the chain rule we have

$$
(K(g(b)-g(t)))^{\prime}=-K^{\prime}(g(b)-g(t)) g^{\prime}(t)=-k(g(b)-g(t)) g^{\prime}(t)
$$

for $t \in(x, b)$ and

$$
(K(g(t)-g(a)))^{\prime}=K^{\prime}(g(t)-g(a)) g^{\prime}(t)=k(g(t)-g(a)) g^{\prime}(t)
$$

for $t \in(a, x)$.

Then we have

$$
\int_{a}^{x} k(g(t)-g(a)) g^{\prime}(t)\left[\frac{t-a}{x-a} f(x)+\frac{x-t}{x-a} f(a)\right] d t
$$




$$
\begin{aligned}
& =\int_{a}^{x}(K(g(t)-g(a)))^{\prime}\left[\frac{t-a}{x-a} f(x)+\frac{x-t}{x-a} f(a)\right] d t \\
& =\left.K(g(t)-g(a))\left[\frac{t-a}{x-a} f(x)+\frac{x-t}{x-a} f(a)\right]\right|_{a} ^{x}-\frac{f(x)-f(a)}{x-a} \int_{a}^{x} K(g(t)-g(a)) d t \\
& =K(g(x)-g(a)) f(x)-\frac{f(x)-f(a)}{x-a} \int_{a}^{x} K(g(t)-g(a)) d t
\end{aligned}
$$

and

$$
\begin{aligned}
& \int_{x}^{b} k(g(b)-g(t)) g^{\prime}(t)\left[\frac{t-x}{b-x} f(b)+\frac{b-t}{b-x} f(x)\right] d t \\
& =-\int_{x}^{b}(K(g(b)-g(t)))^{\prime}\left[\frac{t-x}{b-x} f(b)+\frac{b-t}{b-x} f(x)\right] d t \\
& =-\left[\left.K(g(b)-g(t))\left[\frac{t-x}{b-x} f(b)+\frac{b-t}{b-x} f(x)\right]\right|_{x} ^{b}-\frac{f(b)-f(x)}{b-x} \int_{x}^{b} K(g(b)-g(t)) d t\right] \\
& =K(g(b)-g(x)) f(x)+\frac{f(b)-f(x)}{b-x} \int_{x}^{b} K(g(b)-g(t)) d t
\end{aligned}
$$

for $x \in(a, b)$.

From (2.13) we get

$$
\begin{aligned}
\breve{S}_{k, g, a+, b-} f(x) & \leq \frac{1}{2}\left[K(g(x)-g(a)) f(x)-\frac{f(x)-f(a)}{x-a} \int_{a}^{x} K(g(t)-g(a)) d t\right] \\
& +\frac{1}{2}\left[K(g(b)-g(x)) f(x)+\frac{f(b)-f(x)}{b-x} \int_{x}^{b} K(g(b)-g(t)) d t\right] \\
& =\frac{1}{2}[K(g(x)-g(a))+K(g(b)-g(x))] f(x) \\
& +\frac{1}{2}\left[\frac{f(b)-f(x)}{b-x} \int_{x}^{b} K(g(b)-g(t)) d t-\frac{f(x)-f(a)}{x-a} \int_{a}^{x} K(g(t)-g(a)) d t\right],
\end{aligned}
$$

which proves the third inequality in (2.12).

By Jensen's inequality (2.5) we also have

$$
\begin{aligned}
& \int_{a}^{x} k(g(t)-g(a)) g^{\prime}(t) f(t) d t \\
& \quad \geq f\left(\frac{\int_{a}^{x} k(g(t)-g(a)) g^{\prime}(t) t d t}{\int_{a}^{x} k(g(t)-g(a)) g^{\prime}(t) d t}\right) \int_{a}^{x} k(g(t)-g(a)) g^{\prime}(t) d t
\end{aligned}
$$

and

$$
\begin{aligned}
& \int_{x}^{b} k(g(b)-g(t)) g^{\prime}(t) f(t) d t \\
& \quad \geq f\left(\frac{\int_{x}^{b} k(g(b)-g(t)) g^{\prime}(t) t d t}{\int_{x}^{b} k(g(b)-g(t)) g^{\prime}(t) d t}\right) \int_{x}^{b} k(g(b)-g(t)) g^{\prime}(t) d t
\end{aligned}
$$

for $x \in(a, b)$. 
Observe that

$$
\int_{a}^{x} k(g(t)-g(a)) g^{\prime}(t) d t=\int_{a}^{x}(K(g(t)-g(a)))^{\prime} d t=K(g(x)-g(a))
$$

and

$$
\begin{aligned}
\int_{a}^{x} k(g(t)-g(a)) g^{\prime}(t) t d t & =\int_{a}^{x}(K(g(t)-g(a)))^{\prime} t d t \\
& =\left.K(g(t)-g(a)) t\right|_{a} ^{x}-\int_{a}^{x} K(g(t)-g(a)) d t \\
& =K(g(x)-g(a)) x-\int_{a}^{x} K(g(t)-g(a)) d t
\end{aligned}
$$

for $x \in(a, b)$.

Also

$$
\int_{x}^{b} k(g(b)-g(t)) g^{\prime}(t) d t=-\int_{x}^{b}(K(g(b)-g(t)))^{\prime} d t=K(g(b)-g(x))
$$

and

$$
\begin{aligned}
\int_{x}^{b} k(g(b)-g(t)) g^{\prime}(t) t d t & =-\int_{x}^{b}(K(g(b)-g(t)))^{\prime} t d t \\
& =-\left[\left.K(g(b)-g(t)) t\right|_{x} ^{b}-\int_{x}^{b} K(g(b)-g(t)) d t\right] \\
& =K(g(b)-g(x)) x+\int_{x}^{b} K(g(b)-g(t)) d t
\end{aligned}
$$

for $x \in(a, b)$.

Therefore, by (2.14) and (2.15) we have

$$
\begin{aligned}
\breve{S}_{k, g, a+, b-} f(x)= & \frac{1}{2} \int_{a}^{x} k(g(t)-g(a)) g^{\prime}(t) f(t) d t+\frac{1}{2} \int_{x}^{b} k(g(b)-g(t)) g^{\prime}(t) f(t) d t \\
\geq & \frac{1}{2} f\left(x-\frac{1}{K(g(x)-g(a))} \int_{a}^{x} K(g(t)-g(a)) d t\right) K(g(x)-g(a)) \\
& +\frac{1}{2} f\left(x+\frac{1}{K(g(b)-g(x))} \int_{x}^{b} K(g(b)-g(t)) d t\right) K(g(b)-g(x)),
\end{aligned}
$$

which prove the second inequality in (2.12).

Using the inequality (2.10) for

$$
\alpha=\frac{K(g(x)-g(a))}{2}, \quad \beta=\frac{K(g(b)-g(x))}{2}
$$

and

$$
c=x-\frac{1}{K(g(x)-g(a))} \int_{a}^{x} K(g(t)-g(a)) d t,
$$




$$
d=x+\frac{1}{K(g(b)-g(x))} \int_{x}^{b} K(g(b)-g(t)) d t
$$

we have

$$
\begin{aligned}
& f\left(x-\frac{1}{K(g(x)-g(a))} \int_{a}^{x} K(g(t)-g(a)) d t\right) \frac{K(g(x)-g(a))}{2} \\
& \quad+f\left(x+\frac{1}{K(g(b)-g(x))} \int_{x}^{b} K(g(b)-g(t)) d t\right) \frac{K(g(b)-g(x))}{2} \\
& \geq \frac{1}{2}[K(g(x)-g(a))+K(g(b)-g(x))] f\left(x+\frac{\int_{x}^{b} K(g(b)-g(t)) d t-\int_{a}^{x} K(g(t)-g(a)) d t}{K(g(x)-g(a))+K(g(b)-g(x))}\right),
\end{aligned}
$$

which proves the first inequality in (2.12).

Corollary 2. With the assumptions of Theorem 2, we have

$$
\begin{aligned}
& K\left(\frac{g(b)-g(a)}{2}\right) f\left(M_{g}(a, b)+\frac{\int_{M_{g}(a, b)}^{b} K(g(b)-g(t)) d t-\int_{a}^{M_{g}(a, b)} K(g(t)-g(a)) d t}{2 K\left(\frac{g(b)-g(a)}{2}\right)}\right) \\
& \leq \frac{1}{2}\left[f\left(M_{g}(a, b)-\frac{1}{K\left(\frac{g(b)-g(a)}{2}\right)} \int_{a}^{M_{g}(a, b)} K(g(t)-g(a)) d t\right)\right. \\
& \left.\quad+f\left(M_{g}(a, b)+\frac{1}{K\left(\frac{g(b)-g(a)}{2}\right)} \int_{M_{g}(a, b)}^{b} K(g(b)-g(t)) d t\right)\right] K\left(\frac{g(b)-g(a)}{2}\right) \\
& \leq \breve{S}_{k, g, a+, b-f\left(M_{g}(a, b)\right)} \quad K\left(\frac{g(b)-g(a)}{2}\right) f\left(M_{g}(a, b)\right)+\frac{1}{2} \frac{f(b)-f\left(M_{g}(a, b)\right)}{b-M_{g}(a, b)} \int_{M_{g}(a, b)}^{b} K(g(b)-g(t)) d t \\
& \quad-\frac{1}{2} \frac{f\left(M_{g}(a, b)\right)-f(a)}{M_{g}(a, b)-a} \int_{a}^{M_{g}(a, b)} K(g(t)-g(a)) d t .
\end{aligned}
$$

\section{Applications for generalized Riemann-Liouville fractional integrals}

If we take $k(t)=\frac{1}{\Gamma(\alpha)} t^{\alpha-1}$, where $\Gamma$ is the Gamma function, then

$$
S_{k, g, a+} f(x)=I_{a+, g}^{\alpha} f(x):=\frac{1}{\Gamma(\alpha)} \int_{a}^{x}[g(x)-g(t)]^{\alpha-1} g^{\prime}(t) f(t) d t
$$

for $a<x \leq b$ and

$$
S_{k, g, b-} f(x)=I_{b-, g}^{\alpha} f(x):=\frac{1}{\Gamma(\alpha)} \int_{x}^{b}[g(t)-g(x)]^{\alpha-1} g^{\prime}(t) f(t) d t
$$

for $a \leq x<b$, which are the generalized left- and right-sided Riemann-Liouville fractional integrals of a function $f$ with respect to another function $g$ on $[a, b]$ as defined in [25, p.100]. 
We consider the mixed operators

$$
I_{g, a+, b-}^{\alpha} f(x):=\frac{1}{2}\left[I_{a+, g}^{\alpha} f(x)+I_{b-, g}^{\alpha} f(x)\right]
$$

and

$$
\breve{I}_{g, a+, b-}^{\alpha} f(x):=\frac{1}{2}\left[I_{x+, g}^{\alpha} f(b)+I_{x-, g}^{\alpha} f(a)\right]
$$

for $x \in(a, b)$.

We observe that for $\alpha>0$ we have

$$
K(t)=\frac{1}{\Gamma(\alpha)} \int_{0}^{t} s^{\alpha-1} d s=\frac{t^{\alpha}}{\alpha \Gamma(\alpha)}=\frac{t^{\alpha}}{\Gamma(\alpha+1)}, t \geq 0 .
$$

In what follows we assume that $f:[a, b] \rightarrow \mathbb{R}$ is a convex function on $[a, b]$. Using the inequality (2.1) we get

$$
\begin{aligned}
& \frac{1}{2 \Gamma(\alpha+1)}\left[(g(x)-g(a))^{\alpha}+(g(b)-g(x))^{\alpha}\right] \\
& \quad \times f\left(\frac{(g(x)-g(a))^{\alpha} a+(g(b)-g(x))^{\alpha} b+\int_{a}^{x}(g(x)-g(t))^{\alpha} d t-\int_{x}^{b}(g(t)-g(x))^{\alpha} d t}{(g(x)-g(a))^{\alpha}+(g(b)-g(x))^{\alpha}}\right) \\
& \leq \frac{1}{2 \Gamma(\alpha+1)}\left[f\left(a+\frac{1}{(g(x)-g(a))^{\alpha}} \int_{a}^{x}(g(x)-g(t))^{\alpha} d t\right)(g(x)-g(a))^{\alpha}\right. \\
&\left.+f\left(b-\frac{1}{(g(b)-g(x))^{\alpha}} \int_{x}^{b}(g(t)-g(x))^{\alpha} d t\right)(g(b)-g(x))^{\alpha}\right] \\
& \leq I_{g, a+, b-}^{\alpha} f(x) \\
& \leq \frac{1}{2 \Gamma(\alpha+1)}\left[(g(x)-g(a))^{\alpha} f(a)+(g(b)-g(x))^{\alpha} f(b)\right] \\
&+\frac{1}{2 \Gamma(\alpha+1)}\left[\frac{f(x)-f(a)}{x-a} \int_{a}^{x}(g(x)-g(t))^{\alpha} d t-\frac{f(b)-f(x)}{b-x} \int_{x}^{b}(g(t)-g(x))^{\alpha} d t\right]
\end{aligned}
$$

while from (2.12) we get

$$
\begin{aligned}
& \frac{1}{2 \Gamma(\alpha+1)}\left[(g(x)-g(a))^{\alpha}+(g(b)-g(x))^{\alpha}\right] \\
& \quad \times f\left(x+\frac{\int_{x}^{b}(g(b)-g(t))^{\alpha} d t-\int_{a}^{x}(g(t)-g(a))^{\alpha} d t}{(g(x)-g(a))^{\alpha}+(g(b)-g(x))^{\alpha}}\right) \\
& \leq \frac{1}{2 \Gamma(\alpha+1)}\left[f\left(x-\frac{1}{(g(x)-g(a))^{\alpha}} \int_{a}^{x}(g(t)-g(a))^{\alpha} d t\right)(g(x)-g(a))^{\alpha}\right. \\
& \left.\quad+f\left(x+\frac{1}{(g(b)-g(x))^{\alpha}} \int_{x}^{b}(g(b)-g(t))^{\alpha} d t\right)(g(b)-g(x))^{\alpha}\right] \\
& \leq \breve{I}_{g, a+, b-}^{\alpha} f(x)
\end{aligned}
$$




$$
\begin{aligned}
\leq & \frac{1}{2 \Gamma(\alpha+1)}\left[(g(x)-g(a))^{\alpha}+(g(b)-g(x))^{\alpha}\right] f(x) \\
& +\frac{1}{2 \Gamma(\alpha+1)}\left[\frac{f(b)-f(x)}{b-x} \int_{x}^{b}(g(b)-g(t))^{\alpha} d t-\frac{f(x)-f(a)}{x-a} \int_{a}^{x}(g(t)-g(a))^{\alpha} d t\right](3.4)
\end{aligned}
$$

for $x \in(a, b)$.

Also, by (2.11) and (2.16) we have

$$
\begin{aligned}
& \frac{1}{2^{\alpha} \Gamma(\alpha+1)}(g(b)-g(a))^{\alpha} \\
& \times f\left(\frac{a+b}{2}+\frac{\int_{a}^{M_{g}(a, b)}\left(\frac{g(a)+g(b)}{2}-g(t)\right)^{\alpha} d t-\int_{M_{g}(a, b)}^{b}\left(g(t)-\frac{g(a)+g(b)}{2}\right)^{\alpha} d t}{2^{1-\alpha}(g(b)-g(a))^{\alpha}}\right) \\
\leq & \frac{1}{2^{\alpha+1} \Gamma(\alpha+1)}\left[f\left(a+\frac{1}{\left(\frac{g(b)-g(a)}{2}\right)^{\alpha}} \int_{a}^{M_{g}(a, b)}\left(\frac{g(a)+g(b)}{2}-g(t)\right)^{\alpha} d t\right)\right. \\
& \left.\left.+f\left(b-\frac{g(b)-g(a)}{2}\right)^{\alpha} \int_{M_{g}(a, b)}^{b}\left(g(t)-\frac{g(a)+g(b)}{2}\right)^{\alpha} d t\right)\right](g(b)-g(a))^{\alpha} \\
\leq & I_{g, a+, b-}^{\alpha}\left(M_{g}(a, b)\right) \\
\leq & \frac{1}{2^{\alpha+1} \Gamma(\alpha+1)}[f(a)+f(b)](g(b)-g(a))^{\alpha} \\
& +\frac{1}{2 \Gamma(\alpha+1)} \frac{f\left(M_{g}(a, b)\right)-f(a)}{M_{g}(a, b)-a} \int_{a}^{M_{g}(a, b)}\left(\frac{g(a)+g(b)}{2}-g(t)\right)^{\alpha} d t \\
& -\frac{1}{2 \Gamma(\alpha+1)} \frac{f(b)-f\left(M_{g}(a, b)\right)}{b-M_{g}(a, b)} \int_{M_{g}(a, b)}^{b}\left(g(t)-\frac{g(a)+g(b)}{2}\right)^{\alpha} d t
\end{aligned}
$$

and

$$
\begin{aligned}
& \frac{1}{2^{\alpha} \Gamma(\alpha+1)}(g(b)-g(a))^{\alpha} \\
& \times f\left(M_{g}(a, b)+\frac{\int_{M_{g}(a, b)}^{b}(g(b)-g(t))^{\alpha} d t-\int_{a}^{M_{g}(a, b)}(g(t)-g(a))^{\alpha} d t}{2^{1-\alpha}(g(b)-g(a))}\right) \\
\leq & \frac{1}{2^{\alpha+1} \Gamma(\alpha+1)}\left[f\left(M_{g}(a, b)-\frac{1}{\left(\frac{g(b)-g(a)}{2}\right)^{\alpha}} \int_{a}^{M_{g}(a, b)}(g(t)-g(a))^{\alpha} d t\right)\right. \\
& \left.+f\left(M_{g}(a, b)+\frac{1}{\left(\frac{g(b)-g(a)}{2}\right)^{\alpha}} \int_{M_{g}(a, b)}^{b}(g(b)-g(t))^{\alpha} d t\right)\right](g(b)-g(a))^{\alpha} \\
\leq & \breve{I}_{g, a+, b-}^{\alpha} f\left(M_{g}(a, b)\right) \\
\leq & \frac{1}{2^{\alpha} \Gamma(\alpha+1)}(g(b)-g(a))^{\alpha} f\left(M_{g}(a, b)\right)
\end{aligned}
$$




$$
\begin{aligned}
& +\frac{1}{2 \Gamma(\alpha+1)} \frac{f(b)-f\left(M_{g}(a, b)\right)}{b-M_{g}(a, b)} \int_{M_{g}(a, b)}^{b}(g(b)-g(t))^{\alpha} d t \\
& -\frac{1}{2 \Gamma(\alpha+1)} \frac{f\left(M_{g}(a, b)\right)-f(a)}{M_{g}(a, b)-a} \int_{a}^{M_{g}(a, b)}(g(t)-g(a))^{\alpha} d t .
\end{aligned}
$$

If we take $g(t)=t, t \in[a, b]$ in (3.3) and (3.4), then we get

$$
\begin{aligned}
& \frac{1}{2 \Gamma(\alpha+1)}\left[(x-a)^{\alpha}+(b-x)^{\alpha}\right] f\left(\frac{\frac{a \alpha+x}{\alpha+1}(x-a)^{\alpha}+\frac{x+\alpha b}{\alpha+1}(b-x)^{\alpha}}{(x-a)^{\alpha}+(b-x)^{\alpha}}\right) \\
\leq & \frac{1}{2 \Gamma(\alpha+1)}\left[f\left(\frac{a \alpha+x}{\alpha+1}\right)(x-a)^{\alpha}+f\left(\frac{x+\alpha b}{\alpha+1}\right)(b-x)^{\alpha}\right] \\
\leq & J_{a+, b-}^{\alpha} f(x) \\
\leq & \frac{1}{2 \Gamma(\alpha+1)}\left[(x-a)^{\alpha} f(a)+(b-x)^{\alpha} f(b)\right] \\
& +\frac{1}{2 \Gamma(\alpha+2)}\left[(f(x)-f(a))(x-a)^{\alpha}-(f(b)-f(x))(b-x)^{\alpha}\right]
\end{aligned}
$$

while from (2.12) we get

$$
\begin{aligned}
& \frac{1}{2 \Gamma(\alpha+1)}\left[(x-a)^{\alpha}+(b-x)^{\alpha}\right] f\left(\frac{\frac{\alpha x+a}{\alpha+1}(x-a)^{\alpha}+\frac{b+\alpha x}{\alpha+1}(b-x)^{\alpha}}{(x-a)^{\alpha}+(b-x)^{\alpha}}\right) \\
& \leq \frac{1}{2 \Gamma(\alpha+1)}\left[f\left(\frac{\alpha x+a}{\alpha+1}\right)(x-a)^{\alpha}+f\left(\frac{b+\alpha x}{\alpha+1}\right)(b-x)^{\alpha}\right] \\
& \leq \breve{J}_{a+, b-}^{\alpha} f(x) \\
& \leq \frac{1}{2 \Gamma(\alpha+1)}\left[(x-a)^{\alpha}+(b-x)^{\alpha}\right] f(x) \\
& \quad+\frac{1}{2 \Gamma(\alpha+2)}\left[(f(b)-f(x))(b-x)^{\alpha}-(f(x)-f(a))(x-a)^{\alpha}\right]
\end{aligned}
$$

for $x \in(a, b)$, where

$$
J_{a+, b-}^{\alpha} f(x):=\frac{1}{2}\left[J_{a+}^{\alpha} f(x)+J_{b-}^{\alpha} f(x)\right]
$$

and

$$
\breve{J}_{a+, b-}^{\alpha} f(x):=\frac{1}{2}\left[J_{x+}^{\alpha} f(b)+J_{x-}^{\alpha} f(a)\right]
$$

for $x \in(a, b)$.

If we take $x=\frac{a+b}{2}$ in (3.7) and (3.8), then we get, after required calculations

$$
\begin{aligned}
\frac{(b-a)^{\alpha}}{2^{\alpha} \Gamma(\alpha+1)} f\left(\frac{a+b}{2}\right) & \leq \frac{(b-a)^{\alpha}}{2^{\alpha+1} \Gamma(\alpha+1)}\left[f\left(\frac{(2 \alpha+1) a+b}{2(\alpha+1)}\right)+f\left(\frac{a+(2 \alpha+1) b}{2(\alpha+1)}\right)\right] \\
& \leq J_{a+, b-}^{\alpha} f\left(\frac{a+b}{2}\right) \\
& \leq \frac{(b-a)^{\alpha}}{2^{\alpha} \Gamma(\alpha+2)} f\left(\frac{a+b}{2}\right)+\frac{\alpha(b-a)^{\alpha}}{2^{\alpha} \Gamma(\alpha+2)} \frac{f(a)+f(b)}{2}
\end{aligned}
$$




$$
\leq \frac{(b-a)^{\alpha}}{2^{\alpha} \Gamma(\alpha+1)} \frac{f(a)+f(b)}{2}
$$

and

$$
\begin{aligned}
\frac{(b-a)^{\alpha}}{2^{\alpha} \Gamma(\alpha+1)} f\left(\frac{a+b}{2}\right) & \leq \frac{(b-a)^{\alpha}}{2^{\alpha+1} \Gamma(\alpha+1)}\left[f\left(\frac{(\alpha+2) a+\alpha b}{2(\alpha+1)}\right)+f\left(\frac{\alpha a+(\alpha+2) b}{2(\alpha+1)}\right)\right] \\
& \leq \breve{J}_{a+, b-}^{\alpha} f\left(\frac{a+b}{2}\right) \\
& \leq \frac{\alpha(b-a)^{\alpha}}{2^{\alpha} \Gamma(\alpha+2)} f\left(\frac{a+b}{2}\right)+\frac{(b-a)^{\alpha}}{2^{\alpha} \Gamma(\alpha+2)} \frac{f(b)+f(a)}{2} \\
& \leq \frac{(b-a)^{\alpha}}{2^{\alpha} \Gamma(\alpha+1)} \frac{f(a)+f(b)}{2} .
\end{aligned}
$$

The last inequalities follow by the fact that

$$
f\left(\frac{a+b}{2}\right) \leq \frac{f(b)+f(a)}{2} .
$$

\section{References}

[1] R. P. Agarwal, M.-J. Luo and R. K. Raina, On Ostrowski type inequalities, Fasc. Math., 56 (2016), 5-27.

[2] A. Aglić Aljinović, Montgomery identity and Ostrowski type inequalities for Riemann-Liouville fractional integral, J. Math. 2014, Art. ID 503195, 6 pp.

[3] T. M. Apostol, Mathematical Analysis, Second Edition, Addison-Wesley Publishing Company, 1975.

[4] A. O. Akdemir, Inequalities of Ostrowski's type for $m$ - and $(\alpha, m)$-logarithmically convex functions via Riemann-Liouville fractional integrals, J. Comput. Anal. Appl., 16 (2014), no. 2, 375-383.

[5] G. A. Anastassiou, Fractional representation formulae under initial conditions and fractional Ostrowski type inequalities, Demonstr. Math., 48 (2015), no. 3, 357-378.

[6] G. A. Anastassiou, The reduction method in fractional calculus and fractional Ostrowski type inequalities, Indian J. Math., 56 (2014), No. 3, 333-357.

[7] H. Budak, M. Z. Sarikaya and E. Set, Generalized Ostrowski type inequalities for functions whose local fractional derivatives are generalized s-convex in the second sense, J. Appl. Math. Comput. Mech. 15 (2016), no. 4, $11-21$.

[8] P. Cerone and S. S. Dragomir, Midpoint-type rules from an inequalities point of view, Handbook of analyticcomputational methods in applied mathematics, 135-200, Chapman \& Hall/CRC, Boca Raton, FL, 2000.

[9] S. S. Dragomir, The Ostrowski's integral inequality for Lipschitzian mappings and applications, Comput. Math. Appl., 38 (1999), no. 11-12, 33-37.

[10] S. S. Dragomir, The Ostrowski integral inequality for mappings of bounded variation, Bull. Austral. Math. Soc., 60 (1999), No. 3, 495-508.

[11] S. S. Dragomir, On the midpoint quadrature formula for mappings with bounded variation and applications, Kragujevac J. Math. 22 (2000), 13-19.

[12] S. S. Dragomir, On the Ostrowski's integral inequality for mappings with bounded variation and applications, Math. Ineq. Appl. 4 (2001), No. 1, 59-66. Preprint: RGMIA Res. Rep. Coll. 2 (1999), Art. 7, [0nline: http://rgmia.org/papers/v2n1/v2n1-7.pdf]

[13] S. S. Dragomir, Refinements of the generalised trapezoid and Ostrowski inequalities for functions of bounded variation, Arch. Math. (Basel) 91 (2008), No. 5, 450-460.

[14] S. S. Dragomir, Refinements of the Ostrowski inequality in terms of the cumulative variation and applications, Analysis (Berlin) 34 (2014), No. 2, 223-240. Preprint: RGMIA Res. Rep. Coll. 16 (2013), Art. 29 [Online:http://rgmia.org/papers/v16/v16a29.pdf]. 
[15] S. S. Dragomir, Ostrowski type inequalities for Lebesgue integral: a survey of recent results, Australian J. Math. Anal. Appl., Volume 14, Issue 1, Article 1, 1-287, 2017. [Online http://ajmaa.org/cgi-bin/paper.pl?string=v14n1/V14I1P1.tex] .

[16] S. S. Dragomir, Some inequalities of Hermite-Hadamard type for convex functions and RiemannLiouville fractional integrals, Preprint RGMIA Res. Rep. Coll. 20 (2017), Art. 40. [Online http://rgmia.org/papers/v20/v20a40.pdf] .

[17] S. S. Dragomir, Ostrowski type inequalities for Riemann-Liouville fractional integrals of bounded variation, Hölder and Lipschitzian functions, Preprint RGMIA Res. Rep. Coll. 20 (2017), Art. 48. [Online http://rgmia.org/papers/v20/v20a48.pdf] .

[18] S. S. Dragomir, Ostrowski type inequalities for generalized Riemann-Liouville fractional integrals of functions with bounded variation, RGMIA Res. Rep. Coll., 20 (2017), Art. 58. [Online http://rgmia.org/papers/v20/v20a58.pdf].

[19] S. S. Dragomir, Further Ostrowski and trapezoid type inequalities for the generalized Riemann-Liouville fractional integrals of functions with bounded variation, RGMIA Res. Rep. Coll., 20 (2017), Art. 84. [0nline http://rgmia.org/papers/v20/v20a84.pdf].

[20] S. S. Dragomir, Ostrowski and trapezoid type inequalities for the generalized $k$-g-fractional integrals of functions with bounded variation, RGMIA Res. Rep. Coll., 20 (2017), Art. 111. [Online http://rgmia.org/papers/v20/v20a111.pdf].

[21] S. S. Dragomir, Some inequalities for the generalized $k$-g-fractional integrals of functions under complex boundedness conditions, RGMIA Res. Rep. Coll., 20 (2017), Art. 119. [0nline http://rgmia.org/papers/v20/v20a119.pdf] .

[22] S. S. Dragomir and C. E. M. Pearce, Selected Topics on Hermite-Hadamard Inequalities and Applications, RGMIA Monographs, 2000. [0nline http://rgmia.org/monographs/hermite_hadamard.html] .

[23] A. Guezane-Lakoud and F. Aissaoui, New fractional inequalities of Ostrowski type, Transylv. J. Math. Mech., 5 (2013), no. 2, 103-106

[24] A. Kashuri and R. Liko, Ostrowski type fractional integral inequalities for generalized $(s, m, \varphi)$-preinvex functions, Aust. J. Math. Anal. Appl., 13 (2016), no. 1, Art. 16, 11 pp.

[25] A. Kilbas, H. M. Srivastava and J. J. Trujillo, Theory and Applications of Fractional Differential Equations. North-Holland Mathematics Studies, 204. Elsevier Science B.V., Amsterdam, 2006. xvi+523 pp. ISBN: 978-0444-51832-3; 0-444-51832-0.

[26] M. Kirane, B. T. Torebek, Hermite-Hadamard, Hermite-Hadamard-Fejer, Dragomir-Agarwal and Pachpatte type Inequalities for convex functions via fractional integrals, Preprint arXiv:1701.00092.

[27] M. A. Noor, K. I. Noor and S. Iftikhar, Fractional Ostrowski inequalities for harmonic h-preinvex functions, Facta Univ. Ser. Math. Inform., 31 (2016), No. 2, 417-445

[28] R. K. Raina, On generalized Wright's hypergeometric functions and fractional calculus operators, East Asian Math. J., 21(2)(2005), 191-203.

[29] M. Z. Sarikaya and H. Filiz, Note on the Ostrowski type inequalities for fractional integrals, Vietnam J. Math., 42 (2014), No. 2, 187-190

[30] M.Z. Sarikaya and H. Budak, Generalized Ostrowski type inequalities for local fractional integrals, Proc. Amer. Math. Soc., 145 (2017), No. 4, 1527-1538.

[31] E. Set, New inequalities of Ostrowski type for mappings whose derivatives are s-convex in the second sense via fractional integrals, Comput. Math. Appl., 63 (2012), No. 7, 1147-1154.

[32] M. Tunç, On new inequalities for $h$-convex functions via Riemann-Liouville fractional integration, Filomat, 27:4 (2013), 559-565.

[33] M. Tunç, Ostrowski type inequalities for $m$ - and $(\alpha, m)$-geometrically convex functions via Riemann-Louville fractional integrals, Afr. Mat., 27 (2016), No. 5-6, 841-850.

[34] H. Yildirim and Z. Kirtay, Ostrowski inequality for generalized fractional integral and related inequalities, Malaya J. Mat., 2(3) (2014), 322-329.

[35] C. Yildiz, E, Özdemir and Z. S. Muhamet, New generalizations of Ostrowski-like type inequalities for fractional integrals, Kyungpook Math. J., 56 (2016), No. 1, 161-172. 
[36] H. Yue, Ostrowski inequality for fractional integrals and related fractional inequalities, Transylv. J. Math. Mech., 5 (2013), No. 1, 85-89.

Mathematics, College of Engineering \& Science, Victoria University, PO Box 14428, Melbourne City, MC 8001, Australia.

DST-NRF Centre of Excellence, in the Mathematical and Statistical Sciences, School of Computer Science \& Applied Mathematics, University of the Witwatersrand, Private Bag 3, Johannesburg 2050, South Africa.

E-mail: sever.dragomir@vu.edu.au 